\title{
Robust Control of Planar Dual-Arm Cooperative Manipulators
}

\author{
Jin-Fan Liu \\ LMS Computer Aided Design and Simulation, Inc. (LMS-CADSI) \\ Oakdale, IA 52241 \\ Karim Abdel-Malek ${ }^{1}$ \\ Center for Computer Aided Design and \\ Department of Mechanical Engineering \\ The University of Iowa \\ Iowa City, IA 52242 \\ (319) 335-5676 \\ amalek@engineering.uiowa.edu
}

\begin{abstract}
A robust control method is developed for a planar dual-arm manipulator system. Contact and friction constraints for grasp conditions are considered. An optimization algorithm is developed such that a minimization of the energy consumed by the participating arms subject to equality and inequality constraints of grasp and friction constraints. The necessary KarushKun-Tucker conditions are implemented to characterize admissible solutions. A robust controller is proposed using a switching-sliding algorithm for modeling imprecision and disturbances. The switching-sliding mode is then replaced by a saturation function that results in the elimination of the fundamental cause for control chatter. The formulation presents a control algorithm that is well suited for dual-arm cooperative manipulators.
\end{abstract}

\section{Introduction}

The problem of multiple arm cooperative manipulation has attracted attention in various applications. The potential for a dual arm system in manufacturing and in robot-assisted surgery applications has been contemplated by many researchers (Lee 1989, Montana 1992, Arimoto, et al. 1987, and NASA 1995).

There are many unsolved problems related to dynamic control and grasping of multiple arm cooperation. A near time-optimal inspection-task-sequence planning for two cooperative industrial robots was presented by Cao, et al. (1998) where the algorithm finds a near timeoptimal task sequence of inspection points using continuous joint-acceleration profile. Ahmadabadi and Eiji (1998) introduced the constrain-move concept as a strategy for executing cooperative handling of an object.

\footnotetext{
${ }^{1}$ Corresponding author
} 
Early attempts such as Kerr and Roth (1986), for example, proposed a linear programming approach to consider the intrinsic inequalities of the system which result from manipulatorobject contact criteria. Kumar and Waldron (1988) suggested using interaction forces to address this problem. Using linear programming, Orin and Oh (1981) and Cheng and Orin (1990) suggested minimizing energy consumption in the mechanism. Gardner (1991) and Klein, et al. (1983) addressed the same problem in walking machines. An optimal force distribution scheme of multiple cooperating robots was proposed by Kwon and Lee (1998) that uses the duality theory of nonlinear programming (NLP) combined with the quadratic programming (QP) to treat normal force constraints. Kazerooni (1987) also used NLP methods. Dynamics of cooperating robot systems using the theory of Lie groups was formulated by Ploen and Park (1997). Nakamura, et al. (1989) used a nonlinear programming method to solve this problem. Another approach using Quadratic Programming was implemented by Nahon and Angeles (1992) using an optimization technique to minimize the internal forces in the system. Quadratic programming was demonstrated to be superior to linear programming.

Control strategies of dual robot-arm coordination has also been the subject of many studies. Such a model using a dynamic compensation method was presented by $\mathrm{Gu}$, et al. (1994). Optimal control laws minimizing joint torque loading were discussed by $\mathrm{Hu}$ and Goldenberg (1993a). The same authors proposed an adaptive approach to motion and force control of dual-arm systems in terms of three variable errors (position, control force, and internal force) and was discussed based on the Lyapunov stability theory ( $\mathrm{Hu}$ and Goldenberg 1993b). Contact tasks of dual-arm robots were addressed by Hogan (1988) while object impedance control for dual-arm cooperative manipulators was addressed by Schneider, et al. (1989).

A Lyapunov-based controller that ensures stability for cooperative manipulators was discussed by Yale and Agrawal (1998), where the disturbance torque transmitted to the base of the system by the motion of the manipulators is reduced by altering the order of the reference trajectory polynomial and its coefficients. Compared to the Lyapunov point controller alone, the authors determined that the addition of a fifth-order polynomial reference trajectory leads to superior performance in terms of actuator torque magnitudes and payload repositioning. A flexible dual arm system was built by Pfeffer and Cannon (1993).

Bonitz and Hsia (1996) addressed the minimal internal force required to maintain the grasp on the object is computed from the frictional constraints and sensed forces. A closed-form solution to the minimization problem is developed which makes the algorithm suitable for realtime control. The controller uses sensed moments at the palm interface to maintain proper orientation of the palms to achieve maximum surface contact. Each manipulator's nonlinear dynamics is compensated by a robust auxiliary controller which is insensitive to robot-model uncertainty and payload variation. The controller is only weakly dependent on each manipulator's inertia matrix. Stability of the system is analyzed.

Schneider, et al. (1992) developed a control policy that enforces a controlled impedance not of the individual arm endpoints, but of the manipulated object itself. A parallel implementation for 
a multiprocessor system is presented. The controller fully compensates for the system dynamics and directly controls the object internal forces. Other works of interest in this field include Gouo, et al. (1998), Li and Latombe (1997), Agrawal and Shirumalla (1994), and Tarn, et al. (1996).

In this paper, a methodology for developing a robust controller will be presented. Object dynamics and a proposed control structure are developed in Section 2. Grasp conditions for the dual-arm system on the object are characterized in terms of inequality constraints in Section 3. Robot dynamics expressed in terms of control variables are developed in Section 4. The control of a dual-arm system is formulated as an optimization problem, developed in Section 5 and based on the minimization of the energy cost function. Using the Kuhn-Tucker (K-T) necessary conditions, several cases are addressed and global minimum solutions are expressed based on the K-T switching conditions. The switching-sliding mode is then replaced by a saturation function that results in the elimination of the fundamental cause for control chatter.

\section{Object Dynamics}

Multiple-arm manipulation of objects provides flexibility and versatility in task execution. The advantages, however, are at the expense of increased control complexity due to the additional requirements of maintaining a grasp. Coordinating the dynamic interaction between the participating robot arms also adds to the complexity. The controller of multiple-arm cooperative manipulators should include various characteristics such as a position and force control of the object in free and contact motion. To illustrate the proposed methodology, consider the dual-arm manipulator system holding a rectangular object shown in Fig. 1.

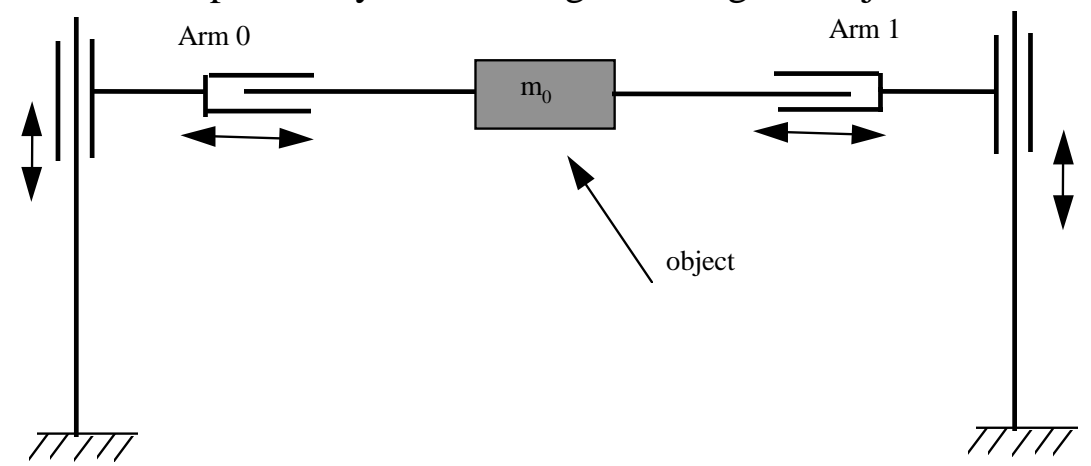

Figure 1 A dual-arm cooperative system

Each arm has two translational joints and the object is assumed to be firmly grasped with a noslip condition. Because the object is firmly grasped, the system has two degrees of freedom. Positions and velocities of the object are determined by measuring joint displacements and velocities of the leader.

The origin of the object reference frame is conveniently located at the task frame of the leader. Note that to successfully measure and control the position of the object, the end-effector of the leader must be in static contact with the object surface throughout the coordinated motion. No relative motion between the task frame of the leader and the object reference frame is allowed. Tactile sensors are assumed to be installed on the fingertips of both arms. 
Consider an object of mass $m_{0}$ being manipulated by the dual-arm system. The free body diagram of $m_{0}$ is shown in Fig. 2. The force supplied by Arm 0 to the object from the $\mathrm{x}-$ direction will be denoted by $f_{a r m 0 x}$, and from the y-direction as $f_{\text {arm } 0 y}$. Similarly the force supplied by Arm 1 to the object from the x-direction is denoted by $f_{\text {armlx }}$, and from the ydirection as $f_{\text {arm } 1}$.

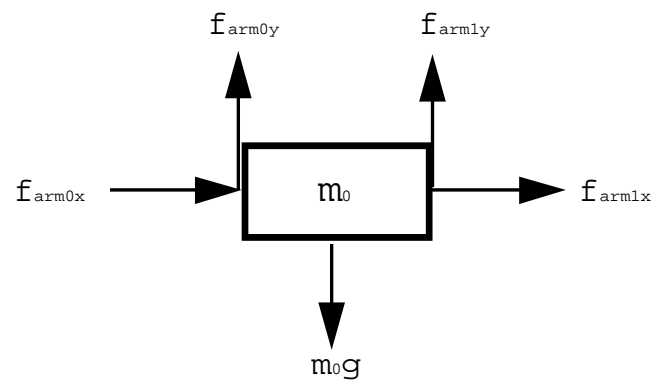

Figure 2 Free Body Diagram of the Object

The Newton-Euler equations of motion of the object in vector form are

$$
\mathbf{M}_{0} \ddot{\mathbf{x}}_{0}+\mathbf{M}_{0} \mathbf{g}=\mathbf{f}_{\text {arm } 0}+\mathbf{f}_{\text {arm } 1}
$$

where $\mathbf{M}_{0}=\left[\begin{array}{cc}m_{0} & 0 \\ 0 & m_{0}\end{array}\right]$ is the mass matrix, $\mathbf{g}=\left[\begin{array}{ll}0 & g\end{array}\right]^{T}$ is the gravity vector, $\ddot{\mathbf{x}}_{0}=\left[\begin{array}{ll}\ddot{x}_{0 x} & \ddot{x}_{0 y}\end{array}\right]^{T}$ is the acceleration vector of the object, and $\mathbf{f}_{\text {arm } 0}=\left[\begin{array}{ll}f_{\text {arm } 0 x} & f_{\text {arm0y }}\end{array}\right]^{T}$ and $\mathbf{f}_{\text {arm } 1}=\left[\begin{array}{ll}f_{\text {arm } 1 x} & f_{\text {arm } 1 y}\end{array}\right]^{T}$ are the forces acting on the contact surface of the object by Arm 0 and Arm 1, respectively. It is assumed that the object will not undergo any rotational motion. Let $\mathbf{f}_{d}$ be the desired force acting on the object by both arms. A control law may be written as

$$
\mathbf{f}_{d}=\mathbf{M}_{0} \mathbf{a}^{\prime}+\mathbf{M}_{0} \mathbf{g}
$$

where the acceleration vector $\mathbf{a}^{\prime}$ is expressed using velocity control constants $\mathbf{K}_{v}$ and proportional constants $\mathbf{K}_{p}$ as

$$
\mathbf{a}^{\prime}=\ddot{\mathbf{x}}_{r}+\mathbf{K}_{v}\left(\dot{\mathbf{x}}_{r}-\dot{\mathbf{x}}_{0}\right)+\mathbf{K}_{p}\left(\dot{\mathbf{x}}_{r}-\dot{\mathbf{x}}_{0}\right)-\mathbf{u}
$$

where $\dot{\mathbf{x}}_{o}$ is the velocity of the object, $\mathbf{u}=\left[\begin{array}{ll}u_{x} & u_{y}\end{array}\right]^{T}$ is a control variable to be determined (Section 6) and $\mathbf{x}_{r}=\left[\begin{array}{ll}x_{r x}(t) & x_{r y}(t)\end{array}\right]^{T}, \dot{\mathbf{x}}_{r}$, and $\ddot{\mathbf{x}}_{r}$ are the given position, velocity, and acceleration of the reference trajectory of $\mathbf{x}_{0}$. Both constants $\mathbf{K}_{v}$ and $\mathbf{K}_{p}$ are $(2 \times 2)$ diagonal matrices with strictly positive elements.

The desired contact force provided by Arm 0 is $\mathbf{f}_{d 0}$ and has components $\mathbf{f}_{d 0}=\left[\begin{array}{ll}f_{d 0 x} & f_{d 0 y}\end{array}\right]^{T}$. The contact force provided by Arm 1 is $\mathbf{f}_{d 1}$ and has components $\mathbf{f}_{d 1}=\left[\begin{array}{ll}f_{d 1 x} & f_{d 1 y}\end{array}\right]^{T}$. The desired resultant force $\mathbf{f}_{d}=\left[\begin{array}{ll}f_{d x} & f_{d y}\end{array}\right]^{T}=\mathbf{f}_{d 0}+\mathbf{f}_{d 1}$ acting on the object can be written as

$$
\mathbf{f}_{d}=\llbracket\left[\begin{array}{lll}
\mathbf{I}_{2} & \mathbf{I}_{2}
\end{array}\right]\left[\begin{array}{ll}
\mathbf{f}_{d 0} & \mathbf{f}_{d 1}
\end{array}\right]^{T} \equiv \mathbf{K} \overline{\mathbf{f}}_{d}
$$


where $\mathbf{I}_{2}$ is a $(2 \times 2)$ identity matrix, $\overline{\mathbf{f}}_{d}=\left[\begin{array}{llll}f_{d 0 x} & f_{d 0 y} & f_{d 1 x} & f_{d 1 y}\end{array}\right]^{T}$, and $\mathbf{K}=\left[\begin{array}{l:l}\mathbf{I}_{2} & \mathbf{I}_{2}\end{array}\right]$ is a $(2 \times 4)$ matrix. Let $\mathbf{K}^{+}$be the pseudo-inverse of $\mathbf{K}$ (Allgower and George 1990), such that $\overline{\mathbf{f}}_{d}$ can be written explicitly as

$$
\overline{\mathbf{f}}_{d}=\mathbf{K}^{+} \mathbf{f}_{d}+\left(\mathbf{I}_{4}-\mathbf{K}^{+} \mathbf{K}\right) \overline{\boldsymbol{\Phi}}_{d}
$$

where we define $\overline{\boldsymbol{\Phi}}_{d} \equiv\left[\begin{array}{ll:ll}\zeta_{d 0 x} & \zeta_{d 0 y} & \zeta_{d 1 x} & \zeta_{d 1 y}\end{array}\right]^{T}=\left[\begin{array}{ll}\boldsymbol{\Phi}_{d 0}^{T} & \boldsymbol{\Phi}_{d 1}^{T}\end{array}\right]^{T}$ as an arbitrary vector and $\mathbf{I}_{4}$ is a $(4 \times 4)$ identity matrix. The pseudo-inverse $\mathbf{K}^{+}$, called the non-squeezing inverse of $\mathbf{K}$ by Walker (1991), is defined by setting it equal to a desired dynamic load distribution matrix $\Lambda_{d}$ (Lu and Meng 1992) such that

$$
\mathbf{K}^{+}=\Lambda_{d}=\left[\begin{array}{l}
\Lambda_{d 0} \\
\Lambda_{d 1}
\end{array}\right]=\left[\begin{array}{cc}
\lambda_{d 0 x} & 0 \\
0 & \lambda_{d 0 y} \\
\hdashline \lambda_{d 1 x} & 0 \\
0 & \lambda_{d 1 y}
\end{array}\right]
$$

Replacing $\mathbf{K}^{+}$by $\boldsymbol{\Lambda}_{d}$ in Eq. (5) yields

$$
\overline{\mathbf{f}}_{d}=\Lambda_{d} \mathbf{f}_{d}+\left(\mathbf{I}_{4}-\Lambda_{d} \mathbf{K}\right) \overline{\boldsymbol{\Phi}}_{d}
$$

Define the second term of the right hand side of Eq. (5) as the internal force $\overline{\mathbf{f}}_{\text {int }}=\left[\begin{array}{ll}f_{\text {int } 0} & f_{\text {int } 0}\end{array}\right]^{T}$ such that $\overline{\mathbf{f}}_{\text {int }}=\left(\mathbf{I}_{4}-\Lambda_{d} \mathbf{K}\right) \overline{\boldsymbol{\Phi}}_{d}$ which spans the null space of $\mathbf{K}$ and does not contribute to the motion of the object. Let

$$
\boldsymbol{\Phi}_{d 0}=-\boldsymbol{\Phi}_{d 1}
$$

then the third term of Eq. (7) $\Lambda_{d} \mathbf{K} \overline{\boldsymbol{\Phi}}_{d}=\mathbf{0}$; i.e., $\overline{\mathbf{f}}_{\text {int }}=\overline{\boldsymbol{\Phi}}_{d}$ and Eq. (7) simplifies to

$$
\overline{\mathbf{f}}_{d}=\Lambda_{d} \mathbf{f}_{d}+\overline{\boldsymbol{\Phi}}_{d}
$$

Equation (9) written separately for Arm 0 and Arm 1 is

and

$$
\begin{aligned}
\mathbf{f}_{d 0} & =\Lambda_{d 0} \mathbf{f}_{d}+\boldsymbol{\Phi}_{d 0} \\
\mathbf{f}_{d 1} & =\Lambda_{d 1} \mathbf{f}_{d}+\boldsymbol{\Phi}_{d 1}
\end{aligned}
$$

Adding Eq. (10) and Eq. (11) and using Eq. (8) yields

$$
\Lambda_{d 0}+\Lambda_{d 1}=\mathbf{I}_{2}
$$

and in terms of the diagonal elements of $\Lambda_{d 0}$ and $\Lambda_{d 1}$ can be written as

$$
\begin{aligned}
& \lambda_{d 0 x}+\lambda_{d 1 x}=1 \\
& \lambda_{d 0 y}+\lambda_{d 1 y}=1
\end{aligned}
$$

Equations (16) and (17) are the first two equality constraints that will be used to formulate the optimization problem. In order to completely define loading conditions, it is necessary to introduce the dynamic load distribution vector denoted by $\mathcal{X}$ as

$$
\boldsymbol{X}=\left[\begin{array}{ll}
\boldsymbol{\chi}_{0}^{T} & \boldsymbol{\chi}_{1}^{T}
\end{array}\right]^{T}=\left[\begin{array}{ll:ll}
\lambda_{d 0 x} & \lambda_{d 0 y} & \lambda_{d 1 x} & \lambda_{d 1 y}
\end{array}\right]^{T}
$$

and define the $(2 \times 2)$ force matrix as

$$
\mathbf{F}=\left[\begin{array}{cc}
f_{d x} & 0 \\
0 & f_{d y}
\end{array}\right]
$$

Equations (11) and (11) can be written as 
and

$$
\begin{aligned}
\mathbf{f}_{d 0} & =\mathbf{f}_{\boldsymbol{X}_{0}}+\boldsymbol{\Phi}_{d 0} \\
\mathbf{f}_{d 1} & =\mathbf{f}_{\boldsymbol{X}_{1}}+\boldsymbol{\Phi}_{d 1}
\end{aligned}
$$

The vector $\boldsymbol{\Phi}_{d i}$ is a function of $\mathbf{x}_{i}-\mathbf{x}_{0}$ and its first derivative. The proper control of $\boldsymbol{\Phi}_{d i}$ is crucial to the grasp-contact stability as was shown by Montana (1992). To simplify expressing the optimum formulation in closed form $\boldsymbol{\Phi}_{d i}$ is assumed to be a given value. It is the desired internal force acting on the contact surface by the $i$ th arm. In order to take into consideration internal grasp forces, friction constraints have to be developed. Figure 3 illustrates the block diagram of the proposed structure of the controller.

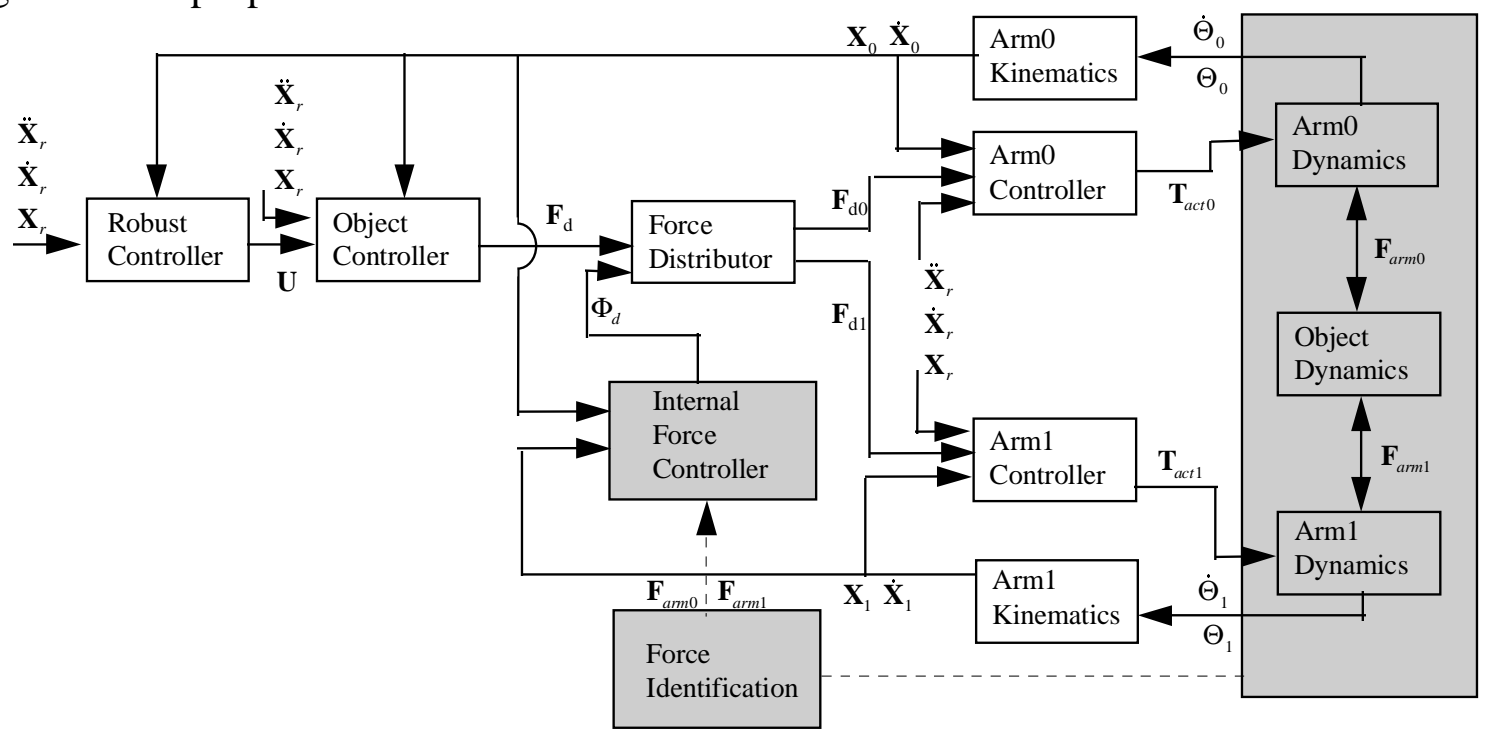

Figure 3 Controller Structure

Contact and frictions constraints on the object characterize grasp conditions and are discussed in the following section.

\section{Grasp Conditions}

To achieve a no-slippage contact grip, the following contact and friction constraints have to be satisfied (Kerr and Roth 1986, Montana 1992).

(1) Contact Constraints:

$$
\begin{aligned}
f_{d 0 x} & \geq 0 \\
f_{d 1 x} & \leq 0
\end{aligned}
$$

Contact forces acting on the object must act along the normal to the contact surface. Equations (19) and (20) can be expressed in terms of the components of $\chi$ as

$$
\begin{gathered}
-f_{d x} \lambda_{d 0 x}-\zeta_{d 0 x} \leq 0 \\
f_{d x} \lambda_{d 1 x}+\zeta_{d 1 x} \leq 0
\end{gathered}
$$

(2) Friction Constraints:

The normal force to the contact surface has to prevent slippage such that

$$
\begin{gathered}
\mu f_{d 0 x} \geq\left|f_{d 0 y}\right| \\
-\mu f_{d 1 x} \geq\left|f_{d 1 y}\right|
\end{gathered}
$$


where $\mu$ is the friction coefficient. The above equations can also be expressed in standard form in terms of the components of the dynamic load distribution vector $\boldsymbol{X}$ such that

$$
\begin{gathered}
-\mu f_{d x} \lambda_{d 0 x}-f_{d y} \lambda_{d 0 y}-\left(\mu \zeta_{d 0 x}+\zeta_{d 0 y}\right) \leq 0 \\
-\mu f_{d x} \lambda_{d 0 x}+f_{d y} \lambda_{d 0 y}-\left(\mu \zeta_{d 0 x}-\zeta_{d 0 y}\right) \leq 0 \\
\mu f_{d x} \lambda_{d 1 x}-f_{d y} \lambda_{d 1 y}+\left(\mu \zeta_{d 1 x}-\zeta_{d 1 y}\right) \leq 0 \\
\mu f_{d x} \lambda_{d 1 x}+f_{d y} \lambda_{d 1 y}+\left(\mu \zeta_{d 1 x}+\zeta_{d 1 y}\right) \leq 0
\end{gathered}
$$

The set of equality constraints Eqs. (13 and 14) and inequalities (21, 22, and 25-28) completely describe the feasible region. Before presenting the problem as a minimization of a cost function $\mathrm{f}_{\text {cost }}$ (Section 6), the kinetics of the dual-arm systems must be addressed.

\section{Robot Dynamics}

The equations of motion for Arm 0 are

$$
\mathbf{H}_{0} \ddot{\boldsymbol{\theta}}_{0}+\mathbf{g}_{0}=\mathbf{t}_{\text {act } 0}-\mathbf{J}_{0}^{T} \mathbf{f}_{\text {arm } 0}
$$

where $\mathbf{H}_{0}=\left[\begin{array}{cc}m_{01}+m_{02} & 0 \\ 0 & m_{02}\end{array}\right]$ is the mass matrix, $\ddot{\boldsymbol{\theta}}_{0}=\left[\begin{array}{l}\ddot{q}_{01} \\ \ddot{q}_{02}\end{array}\right]$ is the acceleration vector in terms of the generalized coordinates of Arm $0\left(q_{01}\right.$ and $\left.\left.q_{02}\right) ; \mathbf{g}_{0}=\llbracket\left(m_{01}+m_{02}\right) g \quad 0\right]^{T}$ is the gravity force vector; $\mathbf{t}_{\text {act } 0}=\left[\begin{array}{ll}\tau_{\text {act } 01} & \tau_{\text {act } 02}\end{array}\right]^{T}$ is the vector of actuating forces for Arm 0 and Arm 1: $\tau_{a c t 01}$ and $\tau_{a c t 02}$, respectively; and $\mathbf{J}_{0}^{T}=\left[\begin{array}{ll}0 & 1 \\ 1 & 0\end{array}\right]$. The actuating force for Arm 0 is $\mathbf{t}_{a c t 0}$ and can be written as

$$
\mathbf{t}_{a c t 0}=\mathbf{t}_{0}^{\prime}+\mathbf{J}_{0}^{T} \mathbf{f}_{d 0}
$$

where $\mathbf{t}_{0}^{\prime}$ can be written as a function of control constants as

$$
\mathbf{t}_{0}^{\prime}=\mathbf{H}_{0} \mathbf{J}_{0}^{-1}\left(\ddot{\mathbf{x}}_{r}-\dot{\mathbf{J}}_{0} \dot{\boldsymbol{\theta}}_{0}\right)+\mathbf{K}_{v}\left(\dot{\mathbf{x}}_{r}-\dot{\mathbf{x}}_{0}\right)+\mathbf{K}_{p}\left(\mathbf{x}_{r}-\mathbf{x}_{0}\right)+\mathbf{g}_{0}
$$

The resulting robot dynamics for Arm 0 in task space becomes

$$
\left(\ddot{\mathbf{x}}_{r}-\ddot{\mathbf{x}}_{0}\right)+\mathbf{K}_{v}\left(\dot{\mathbf{x}}_{r}-\dot{\mathbf{x}}_{0}\right)+\mathbf{K}_{p}\left(\mathbf{x}_{r}-\mathbf{x}_{0}\right)=\mathbf{J}_{0} \mathbf{H}_{0}^{-1} \mathbf{J}_{0}^{T}\left(\mathbf{f}_{a r m 0}-\mathbf{f}_{d 0}\right)
$$

Forces acting on the two arms are depicted in Fig. 4.

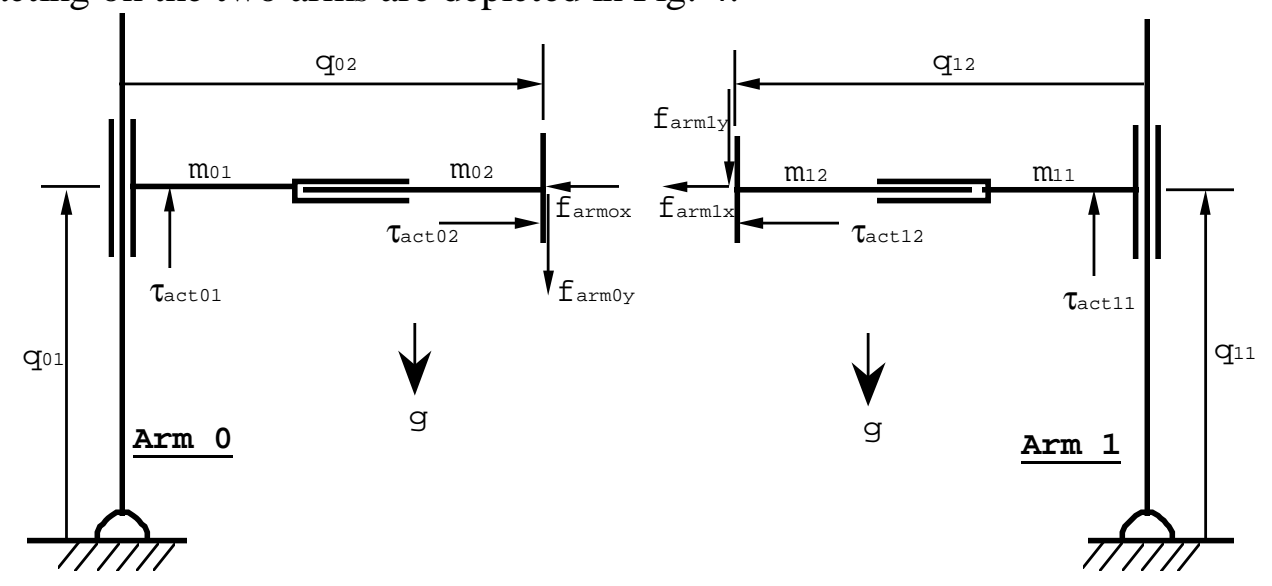

Figure 4 Free Body Diagram of Arm 0 and Arm 1

Similarly, the equations of motion for Arm 1 are: 
$\mathbf{H}_{1} \ddot{\boldsymbol{\theta}}_{1}+\mathbf{g}_{1}=\mathbf{t}_{a c t 1}-\mathbf{J}_{1}^{T} \mathbf{f}_{a r m 1}$
where $\mathbf{H}_{1}=\left[\begin{array}{cc}m_{11}+m_{12} & 0 \\ 0 & m_{12}\end{array}\right], \ddot{\boldsymbol{\theta}}_{1}=\left[\begin{array}{l}\ddot{q}_{11} \\ \ddot{q}_{12}\end{array}\right], \mathbf{g}_{1}=\left[\begin{array}{c}\left(m_{11}+m_{12}\right) g \\ 0\end{array}\right], \mathbf{t}_{a c t 1}=\left[\begin{array}{l}\tau_{a c t 11} \\ \tau_{a c t 12}\end{array}\right]$, and

$\mathbf{J}_{1}^{T}=\left[\begin{array}{cc}0 & 1 \\ -1 & 0\end{array}\right]$ and the actuating forces for Arm $1\left(\mathbf{t}_{a c t 1}\right)$ are

$$
\mathbf{t}_{a c t 1}=\mathbf{t}_{1}^{\prime}+\mathbf{J}_{1}^{T} \mathbf{f}_{d 1}
$$

where

$$
\mathbf{t}_{1}^{\prime}=\mathbf{H}_{1} \mathbf{J}_{1}^{-1}\left(\ddot{\mathbf{X}}_{r}-\dot{\mathbf{J}}_{1} \dot{\boldsymbol{\theta}}_{1}\right)+\mathbf{K}_{v}\left(\dot{\mathbf{x}}_{r}-\dot{\mathbf{x}}_{1}\right)+\mathbf{K}_{p}\left(\mathbf{x}_{r}-\mathbf{x}_{1}\right)+\mathbf{g}_{1}
$$

Robot dynamics for Arm 1 in task space becomes

$$
\left(\ddot{\mathbf{x}}_{r}-\ddot{\mathbf{x}}_{1}\right)+\mathbf{K}_{v}\left(\dot{\mathbf{x}}_{r}-\dot{\mathbf{x}}_{1}\right)+\mathbf{K}_{p}\left(\mathbf{x}_{r}-\mathbf{x}_{1}\right)=\mathbf{J}_{1} \mathbf{H}_{1}^{-1} \mathbf{J}_{1}^{T}\left(\mathbf{f}_{a r m 1}-\mathbf{f}_{d 1}\right)
$$

Substituting Eq. (32) and Eq. (36) into Eq. (2) and rearranging yields

$$
\begin{aligned}
& \left(\ddot{\mathbf{x}}_{r}-\ddot{\mathbf{x}}_{0}\right)+\mathbf{K}_{v}\left(\dot{\mathbf{x}}_{r}-\dot{\mathbf{x}}_{0}\right)+\mathbf{K}_{p}\left(\mathbf{x}_{r}-\mathbf{x}_{0}\right) \\
& =\mathbf{u}-\mathbf{M}_{0}^{-1}\left\{\mathbf{J}_{0}^{-T} \mathbf{H}_{0} \mathbf{J}_{0}^{-1}\left[\left(\ddot{\mathbf{x}}_{r}-\ddot{\mathbf{x}}_{0}\right)+\mathbf{K}_{v}\left(\dot{\mathbf{x}}_{r}-\dot{\mathbf{x}}_{0}\right)+\mathbf{K}_{p}\left(\mathbf{x}_{r}-\mathbf{x}_{0}\right)\right]+\right. \\
& \quad \mathbf{J}_{1} \mathbf{H}_{1}^{-1} \mathbf{J}_{1}^{T}\left[\left(\ddot{\mathbf{x}}_{r}-\ddot{\mathbf{x}}_{1}\right)+\mathbf{K}_{v}\left(\dot{\mathbf{x}}_{r}-\dot{\mathbf{x}}_{1}\right)+\mathbf{K}_{p}\left(\mathbf{x}_{r}-\mathbf{x}_{1}\right)\right]
\end{aligned}
$$

The set of equations (Eq. 37) are the overall equations of motion for the cooperative system expressed in task space. By properly designing a robust control law for $\mathbf{u}$, the left-hand side of Eq. (40) approaches zero, then the value of $\mathbf{f}_{\text {arm } 0}$ approaches the value of $\mathbf{f}_{d 0}$, the value of $\mathbf{f}_{\text {arm } 1}$ approaches the value of $\mathbf{f}_{d 1}$, and $\mathbf{x}_{1}-\mathbf{x}_{0}$ approaches a constant vector as time approaches infinity and the convergence rate can be modulated. The development of a robust controller will be discussed in section 8 .

\section{Minimization of the Energy Cost Function and the Kuhn-Tucker Conditions}

Suppose that the objective in determining an optimal force distribution is to minimize the total energy $E$ consumed by the actuators of the participating arms. It is then required to minimize

where $\quad \overline{\mathbf{t}}_{a c t}^{T} \equiv\left[\begin{array}{ll}\mathbf{t}_{a c t 0}^{T} & \mathbf{t}_{a c t 1}^{T}\end{array}\right]^{T}=\left[\begin{array}{ll:ll}\tau_{a c t 01} & \tau_{a c t 02} & \tau_{a c t 11} & \tau_{a c t 12}\end{array}\right]^{T}$

Equation (38) can be written as

$$
E=\frac{1}{2} \sum_{i=0}^{1} \mathbf{t}_{\text {acti }}^{T} \mathbf{t}_{\text {acti }}
$$

Substituting Eq. (33) and Eq. (34) into Eq. (40) yields

$$
E=\frac{1}{2}\left[\mathbf{t}_{i}^{\prime}+\mathbf{J}_{i}^{T} \mathbf{f}_{d i}\right]^{T}\left[\mathbf{t}_{i}^{\prime}+\mathbf{J}_{i}^{T} \mathbf{f}_{d i}\right] \quad i=0,1
$$

Substituting Eq. (17) and Eq. (18) into Eq. (41), the cost function $\mathrm{f}_{\text {cost }}$ may be defined by collecting the dynamic load distribution terms such that

$$
\mathrm{f}_{\mathrm{costi}}\left(\boldsymbol{\chi}_{i}\right) \equiv\left(\mathbf{f}^{T} \mathbf{J}_{i} \mathbf{t}_{i}^{\prime}+\mathbf{f}^{T} \mathbf{J}_{i} \mathbf{J}_{i}^{T} \boldsymbol{\Phi}_{d i}\right)^{T} \boldsymbol{\chi}_{i}+\frac{1}{2} \boldsymbol{\chi}_{i}^{T}\left(\mathbf{f}^{T} \mathbf{J}_{i} \mathbf{J}_{i}^{T}\right) \boldsymbol{\chi}_{i} \quad i=0,1
$$

or written in terms of two components as 


$$
\mathrm{f}_{\text {cost }}=\Gamma_{0}^{T} \boldsymbol{X}_{0}+\frac{1}{2} \boldsymbol{\chi}_{0}^{T} \boldsymbol{\Psi}_{0} \boldsymbol{X}_{0}+\Gamma_{1}^{T} \boldsymbol{X}_{1}+\frac{1}{2} \boldsymbol{\chi}_{1}^{T} \boldsymbol{\Psi}_{1} \boldsymbol{\chi}_{1}
$$

where

$$
\begin{gathered}
\Gamma_{i}=\mathbf{f}^{T} \mathbf{J}_{i} \mathbf{t}_{i}^{\prime}+\mathbf{f}^{T} \mathbf{J}_{i} \mathbf{J}_{i}^{T} \boldsymbol{\Phi}_{d i} \\
\boldsymbol{\Psi}_{i}=\mathbf{f}^{T} \mathbf{J}_{i} \mathbf{J}_{i}^{T} \mathbf{f}
\end{gathered}
$$

Let $\ddot{\mathbf{x}}_{r}=\mathbf{0}$ and assume that a proper robust control law has been applied on the system, then it is reasonable to expect that $\dot{\mathbf{x}}_{r}-\dot{\mathbf{x}}_{1} \approx \mathbf{0}$ and $\mathbf{x}_{r}-\mathbf{x}_{1} \approx \mathbf{0}$. Then $\mathbf{t}_{i}^{\prime}$ is expressed as

$$
\mathbf{t}_{0}^{\prime}=\mathbf{g}_{0}=\left[\begin{array}{c}
\left(m_{01}+m_{02}\right) g \\
0
\end{array}\right] \text { and } \quad \mathbf{t}_{1}^{\prime}=\mathbf{g}_{1}=\left[\begin{array}{c}
\left(m_{11}+m_{12}\right) g \\
0
\end{array}\right]
$$

Substituting for $\mathbf{t}_{i}^{\prime}$ and $\mathbf{J}_{i}$ into Eq. (49) yields

and

$$
\begin{gathered}
\boldsymbol{\Gamma}_{0}=\left[\begin{array}{c}
\zeta_{d 0 x} f_{d x} \\
\left(\zeta_{d 0 y}+\left(m_{01}+m_{02}\right) g\right) f_{d y}
\end{array}\right] \text { and } \boldsymbol{\Gamma}_{1}=\left[\begin{array}{c}
\zeta_{d 1 x} f_{d x} \\
\left(\zeta_{d 1 y}+\left(m_{11}+m_{12}\right) g\right) f_{d y}
\end{array}\right] \\
\Psi_{0}=\Psi_{1}=\left[\begin{array}{cc}
f_{d x}^{2} & 0 \\
0 & f_{d y}^{2}
\end{array}\right]
\end{gathered}
$$

The cost function is expanded as

$$
\begin{gathered}
\mathrm{f}_{\text {cost }}(\chi)=\frac{1}{2} f_{d x}^{2} \lambda_{d 0 x}^{2}+\zeta_{d 0 x} f_{d x} \lambda_{d 0 x}+\left[\zeta_{d 0 y}+\left(m_{01}+m_{02}\right) g\right] f_{d y} \lambda_{d 0 y}+\frac{1}{2} f_{d y}^{2} \lambda_{d 0 y}^{2} \\
+\frac{1}{2} f_{d x}^{2} \lambda_{d 1 x}^{2}+\zeta_{d 1 x} f_{d x} \lambda_{d 1 x}+\left[\zeta_{d 1 y}+\left(m_{11}+m_{12}\right) g\right] f_{d y} \lambda_{d 1 y}+\frac{1}{2} f_{d y}^{2} \lambda_{d 1 y}^{2}
\end{gathered}
$$

subject to equality constraints Eqs. $(13,14)$, and inequality constraints Eqs. $(21,22)$, and Eqs. (25-28). Rewritten here for clarity, the complete set of constraints denoted by $h_{i}$ is

$$
\begin{gathered}
h_{1} \equiv \lambda_{d 0 x}+\lambda_{d 1 x}-1=0 \\
h_{2} \equiv \lambda_{d 0 y}+\lambda_{d 1 y}-1=0 \\
h_{3} \equiv-f_{d x} \lambda_{d 0 x}-\zeta_{d 0 x} \leq 0 \\
h_{4} \equiv f_{d x} \lambda_{d 1 x}+\zeta_{d 0 x} \leq 0 \\
h_{5} \equiv-\mu f_{d x} \lambda_{d 0 x}-f_{d y} \lambda_{d 0 y}-\left(\mu \zeta_{d 0 x}+\zeta_{d 0 y}\right) \leq 0 \\
h_{6} \equiv-\mu f_{d x} \lambda_{d 0 x}+f_{d y} \lambda_{d 0 y}-\left(\mu \zeta_{d 0 x}-\zeta_{d 0 y}\right) \leq 0 \\
h_{7} \equiv \mu f_{d x} \lambda_{d 1 x}-f_{d y} \lambda_{d 1 y}+\left(\mu \zeta_{d 1 x}-\zeta_{d 1 y}\right) \leq 0 \\
h_{8} \equiv \mu f_{d x} \lambda_{d 1 x}+f_{d y} \lambda_{d 1 y}+\left(\mu \zeta_{d 1 x}+\zeta_{d 1 y}\right) \leq 0
\end{gathered}
$$

The optimization problem is now in standard form and can be stated as a minimization of the cost function such that

$$
\min \mathrm{f}_{\text {cost }}(\boldsymbol{\chi})=\Gamma^{T} \boldsymbol{X}+\frac{1}{2} \boldsymbol{\chi}^{T} \boldsymbol{\Psi} \boldsymbol{\chi}
$$

where $\Gamma=\left[\begin{array}{cc}\Gamma_{0}^{T} & \Gamma_{1}^{T}\end{array}\right]^{T}$ and $\Psi=\left[\begin{array}{cc}\Psi_{0} & \mathbf{0} \\ \mathbf{0} & \boldsymbol{\Psi}_{1}\end{array}\right]=\operatorname{diag}\left(f_{d x}^{2} \quad f_{d y}^{2} \quad f_{d x}^{2} \quad f_{d y}^{2}\right)$ subject to equality and inequality constraints as $h_{i}(\chi)=0 ; i=1,2$ and $h_{i}(\chi) \leq 0 ; i=3, \ldots, 8$. Because both equality and inequality constraints are linear, the feasible region is a convex set. It can be proved that 
the cost function is also a convex function by showing that the Hessian matrix is positive semidefinite (has all eigenvalues nonnegative) at all points in the constraint set, i.e.

$$
\nabla^{2} \mathrm{f}_{\text {cost }}(\chi)=\Psi
$$

has eigenvalues $f_{d x}^{2}, f_{d y}^{2}, f_{d x}^{2}, f_{d y}^{2} \geq 0$. The convexity guarantees that Karush-Kuhn-Tucker condition (Arora 1989) for a regular point in the constraint set is a necessary and sufficient condition. Furthermore, any candidate points for local minimum is also a global minimum.

However, uniqueness of global minimum points is not guaranteed, because the Hessian may not be positive definite (either $f_{d x}$ or $f_{d y}$ could be zero.) In the following discussion, it is assumed that $f_{d x} \cdot f_{d y} \neq 0$. If either $f_{d x}$ or $f_{d y}$ becomes zero, the problem can be treated as a special case and trivially solved (if both become zero, there is no cost function.) Under this assumption, the problem is strictly convex and the K-K-T conditions are both necessary and sufficient for a global minimum point. Note that the regularity of the candidate point has to be checked if more than one constraint is imposed. Regularity is checked by ensuring the linear independence of the set of gradient vectors of active constraints.

In order to determine a solution for the optimization problem, the Lagrangian function $L(\boldsymbol{X}, \mathbf{v})$ is formulated as

$$
L(\boldsymbol{X}, \mathbf{v}) \equiv \mathrm{f}_{\text {cost }}+\mathbf{v}^{T} \mathbf{h}(\chi)
$$

where $\mathbf{v}=\llbracket v_{1}, \ldots, v_{8} \rrbracket^{T}$ are the Lagrange multipliers and $\mathbf{h}(\boldsymbol{\chi})=\llbracket h_{1}, \ldots, h_{8} \rrbracket^{T}$ are the constraints defined above. Consider the K-K-T conditions for a regular point of the constraint set such as

$$
\nabla \mathbf{L}=\nabla \mathrm{f}_{\text {cost }}+(\nabla \mathbf{h}) \mathbf{v}^{T}=\mathbf{0}
$$

subject to the eight constraints and to the switching conditions given by

$$
v_{i} h_{i}(\boldsymbol{X})=0 \quad i=3 . .8
$$

where a non-negativity condition is imposed on Lagrange multiplier of the inequality constraints as

$$
v_{i} \geq 0 \quad i=3 . .8
$$

Since there are six inequality constraints the switching conditions lead to $2^{6}=64$ distinct normal solution cases. If the maximum actuating torque constraints were included, there could have been more distinct solution cases. The method of Goldfarb and Idnani (1983) or the method of Nahon and Angeles (1992a and 1992b) can be applied for strictly convex problems with large numbers of inequality constraints by successively choosing sets of active constraints and minimizing the objective function subject to these constraints.

In order to explicitly solve the K-K-T condition, the problem is simplified by neglecting some of the constraints. In this case, the friction constraints $h_{5}$ through $h_{8}$ can be neglected by assuming that the surfaces of the object and end-effector have matching teeth. The K-K-T condition for the simplified problem can be written as 


$$
\nabla \mathbf{L}=\left[\begin{array}{c}
f_{d x}^{2} \lambda_{d 0 x}+\zeta_{d 0 x} f_{d x}+u_{1}-f_{d x} u_{3} \\
{\left[\zeta_{d 0 x}+\left(m_{01}+m_{02}\right) g\right] f_{d y}+f_{d y}^{2} \lambda_{d 0 y}+u_{2}} \\
f_{d x}^{2} \lambda_{d 1 x}+\zeta_{d 1 x} f_{d x}+u_{1}+f_{d x} u_{4} \\
{\left[\zeta_{d 1 x}+\left(m_{11}+m_{12}\right) g\right] f_{d y}+f_{d y}^{2} \lambda_{d 1 y}+u_{2}}
\end{array}\right]=\mathbf{0}
$$

subject to the following constraints $h_{1}, h_{2}, h_{3}, h_{4}$, the switching conditions

$$
v_{3} h_{3}=0 \text { and } v_{4} h_{4}=0
$$

where $h_{3} \equiv-f_{d x} \lambda_{d 0 x}-\zeta_{d 0 x} \leq 0$ and $h_{4} \equiv f_{d x} \lambda_{d 1 x}+\zeta_{d 0 x} \leq 0$ and the non-negativity condition of the Lagrange multipliers

$$
v_{3} \geq 0, v_{4} \geq 0
$$

Note that the column vectors of the gradient of constraints is

$$
\nabla \mathbf{h}=\left[\begin{array}{cccc}
1 & 0 & -f_{d x} & 0 \\
0 & 1 & 0 & 0 \\
1 & 0 & 0 & f_{d x} \\
0 & 1 & 0 & 0
\end{array}\right]
$$

and is linearly independent if $f_{d x}$ is nonzero. Therefore, all the points in the constraint set are regular if $f_{d x} \neq 0$. The switching conditions in Eqs. (66) give rise to four cases for the solution of the K-K-T conditions. Each case will be separately discussed.

Case 1: $v_{3}=0, v_{4}=0$. Solving the system of Eqs. (64-67) subject to this condition, it can be shown that $h_{3}=-f_{d x} / 2$ and $h_{4}=f_{d x} / 2$. Since both of these conditions cannot satisfy inequalities $h_{3}$ and $h_{4}$ (note that $f_{d x} f_{d y}$ is nonzero), the K-K-T conditions are violated. Thus, there is no solution for this case.

Case 2: $v_{3}=0, h_{4}=0$. It can be shown that $h_{3}=-f_{d x}$ and $v_{4}=f_{d x}$, thus, a solution exists if $f_{d x} \geq 0$ which results in the global minimum point as

$$
\begin{gathered}
\lambda_{d 0 x}=1-\frac{\zeta_{d 0 x}}{f_{d x}} \\
\lambda_{d 1 x}=-\frac{\zeta_{d 1 x}}{f_{d x}} \\
\lambda_{d 0 y}=\frac{1}{2}-\frac{\zeta_{d 0 x}}{f_{d y}}+\frac{\left(m_{11}+m_{12}-m_{01}-m_{02}\right) g}{2 f_{d y}} \\
\lambda_{d 1 y}=\frac{1}{2}-\frac{\zeta_{d 1 x}}{f_{d y}}-\frac{\left(m_{11}+m_{12}-m_{01}-m_{02}\right) g}{2 f_{d y}} \\
v_{2}=-\frac{f_{d y}^{2}}{2}-\frac{\left(m_{11}+m_{12}-m_{01}-m_{02}\right) g f_{d y}}{2} \\
v_{4}=f_{d x}
\end{gathered}
$$


Case 3: $v_{4}=0, h_{3}=0$. It can be shown that $v_{3}=-f_{d x}$ and $h_{4}=f_{d x}$. Thus, a solution exists if $f_{d x} \leq 0$ and the global minimum point is

$$
\begin{gathered}
\lambda_{d 0 x}=-\frac{\zeta_{d 0 x}}{f_{d x}} \\
\lambda_{d 1 x}=1-\frac{\zeta_{d 1 x}}{f_{d x}} \\
\lambda_{d 0 y}=\frac{1}{2}-\frac{\zeta_{d 0 x}}{f_{d y}}+\frac{\left(m_{11}+m_{12}-m_{01}-m_{02}\right) g}{2 f_{d y}} \\
\lambda_{d 1 y}=\frac{1}{2}-\frac{\zeta_{d 1 x}}{f_{d y}}-\frac{\left(m_{11}+m_{12}-m_{01}-m_{02}\right) g}{2 f_{d y}} \\
v_{1}=-f_{d x}^{2} \\
v_{2}=-\frac{f_{d y}^{2}}{2}-\frac{\left(m_{11}+m_{12}-m_{01}-m_{02}\right) g f_{d y}}{2} \\
v_{3}=-f_{d x}
\end{gathered}
$$

Case 4: $h_{3}=0, h_{4}=0$. Solving the system of Eqs. (64-67) and substituting into the inequality constraints results in two violated equality constraints $h_{1}$ and $h_{2}$. The K-K-T conditions are violated, thus, no solution exists for this case.

\section{Robust Controller}

A sliding control algorithm that plays the role of regulating the error given in Eq. (40) will be introduced in this section. First, consider decoupling Eq. (37) along each direction of the endeffector directions and rewriting it as

$$
\mathbf{K}_{p}\left(\mathbf{x}_{r}-\mathbf{x}\right)+\mathbf{K}_{v}\left(\dot{\mathbf{x}}_{r}-\dot{\mathbf{x}}\right)+\left(\ddot{\mathbf{x}}_{r}-\ddot{\mathbf{x}}\right)=\mathbf{u}+\tilde{\mathbf{u}}
$$

where $\widetilde{\mathbf{u}}$ is a vector of perturbation parameters. The goal is to make the left hand side of Eq. (82) equal to zero for a target reference trajectory $\left(\mathbf{x}_{t}\right)$, such that

$$
\mathbf{K}_{p}\left(\mathbf{x}_{r}-\mathbf{x}_{t}\right)+\mathbf{K}_{v}\left(\dot{\mathbf{x}}_{r}-\dot{\mathbf{x}}_{t}\right)+\left(\ddot{\mathbf{x}}_{r}-\ddot{\mathbf{x}}_{t}\right)=\mathbf{0}
$$

where $\mathbf{x}_{t}$ is defined as the Target Impedance Reference Trajectory (TIRT). Subtracting Eq. (83) from Eq. (82) to determine $\mathbf{x}_{r}$ and its derivatives yields

$$
\mathbf{K}_{p}\left(\mathbf{x}_{t}-\mathbf{x}\right)+\mathbf{K}_{v}\left(\dot{\mathbf{x}}_{t}-\dot{\mathbf{x}}\right)+\left(\ddot{\mathbf{x}}_{t}-\ddot{\mathbf{x}}\right)=\mathbf{u}+\tilde{\mathbf{u}}
$$

The error is defined as

$$
\mathbf{e}_{t}=\mathbf{x}_{t}-\mathbf{x}
$$

where $\mathbf{e}_{t}=\llbracket\left[\begin{array}{ll}e_{t 0} & e_{t 1}\end{array}\right]^{T}$. Substituting for $\left(\mathbf{x}_{t}-\mathbf{x}\right)$ into Eq. (84) and rearranging, the resulting error dynamic equation can be written as

$$
\ddot{\mathbf{e}}_{t}=-\mathbf{K}_{p} \mathbf{e}_{t}-\mathbf{K}_{v} \dot{\mathbf{e}}_{t}+\mathbf{u}+\widetilde{\mathbf{u}}
$$

Assume that the sliding surface is chosen as

$$
s_{i}=\dot{e}_{t i}+B_{i} e_{t i}+C_{i} \int_{0}^{t} e_{t i} d \tau \quad i=0,1
$$


where $\mathbf{s}=\left[\begin{array}{ll}s_{0} & s_{1}\end{array}\right]^{T}$ and $\left(B_{i}\right.$ and $\left.C_{i}\right)$ are design parameters that must satisfy the RouthHurwitz criterion (Raven 1995) to form a sliding surface and $\tau$ is time. The vector differentiation of both sides of Eq. (87) yields

$$
\dot{s}_{i}=\ddot{e}_{t}+B_{i} \dot{e}_{t}+C_{i} e_{t} \quad i=0,1
$$

Substitute Eq. (103) into Eq. (105) yields

$$
\dot{s}_{i}=u_{i}+\tilde{u}_{i}-\left(K_{p}\right)_{i i} e_{t i}-\left(K_{v}\right)_{i i} \dot{e}_{t}+B_{i} \dot{e}_{t i}+C_{i} e_{t} i=0,1
$$

where the double indices are used to indicate matrix notation. To attract any given initial error states into the sliding surface, the controller should satisfy the following sliding condition

$$
s_{i} \dot{s}_{i} \leq-\eta_{i}\left|s_{i}\right| \quad i=0,1
$$

where $\eta$ is a positive scalar that can be selected to achieve the desired attracting speed. By letting $\dot{\mathbf{s}}=\mathbf{0}$, the control variable $\mathbf{u}$ can be determined from Eq. (89) such that

$$
u_{i}=\left(\left(K_{p}\right)_{i i}-C_{i}\right) e_{t i}+\left(\left(K_{v}\right)_{i i}-B_{i}\right) \dot{e}_{t i}-\tilde{u}_{i} ; \quad i=0,1
$$

The perturbation vector $\widetilde{\mathbf{u}}$ is an unknown quantity. It is assumed, however, that $\widetilde{\mathbf{u}}$ has a known upper-bound denoted by $\tilde{\mathbf{u}}^{u b}$ such that

$$
|\widetilde{\mathbf{u}}| \leq \widetilde{\mathbf{u}}^{u b}
$$

Consider replacing the unknown perturbation term $\tilde{\mathbf{u}}$ by a sign function $\rho$ such that

$$
u_{i}=\left(\left(K_{p}\right)_{i i}-C_{i}\right) e_{t i}+\left(\left(K_{v}\right)_{i i}-B_{i}\right) \dot{e}_{t i}-\rho_{i} \quad i=0,1
$$

where $\rho=\left[\begin{array}{ll}\rho_{0} & \rho_{1}\end{array}\right]^{T}$ is defined as

$$
\boldsymbol{\rho}=\left(\widetilde{\mathbf{u}}^{u b}+\boldsymbol{\eta}\right) \operatorname{sgn}(\mathbf{S})=\left\{\begin{array}{ccc}
\widetilde{\mathbf{u}}^{u b}+\boldsymbol{\eta} & \text { if } \quad \mathbf{s}>0 \\
\left(\widetilde{\mathbf{u}}^{u b}+\boldsymbol{\eta}\right) & \text { if } \quad \mathbf{s}<0 \\
0 & \text { if } & \mathbf{s}=0
\end{array}\right.
$$

The resulting $\mathbf{s}$ dynamics equation for the exact switching sliding mode control law is

$$
\dot{\mathbf{s}}=\widetilde{\mathbf{u}}-\boldsymbol{\rho}=\widetilde{\mathbf{u}}-\left(\tilde{\mathbf{u}}^{u b}+\boldsymbol{\eta}\right) \operatorname{sgn}(\mathbf{s})
$$

Multiplying both sides if Eq. (95) by $s_{i}$ yields

$$
s_{i} \dot{s}_{i}=s_{i} \tilde{u}_{i}-\left(\tilde{u}_{i}^{u b}+\eta_{i}\right)\left|s_{i}\right|=s_{i} \tilde{u}_{i}-\tilde{u}_{i}^{u b}\left|s_{i}\right|-\eta_{i}\left|s_{i}\right|<0 ; \quad i=0,1
$$

which satisfies the sliding condition of Eq. (90).

Although the above exact switching sliding mode control law is able to compensate for modeling imprecision and disturbances, it includes the sign function $\rho$ and hence has to be discontinuous across $\mathbf{s}(t)$. Since $\rho_{i}$ is discontinuous at $s_{i}=0$, chattering phenomenon may occur in the neighborhood of the sliding surface. A replacement for the sign function by the unit saturation function is proposed by Slotine and Sastry (1983). This operation eliminates the fundamental cause of control chatter by deploying a continuous switching element. However, if the forced oscillations of the $s$-dynamics display high frequencies, the corresponding saturation function control component shows equally high frequency dither, which is not desired either (Elmali 1992). Therefore, it should be advantageous to enforce the $s$-dynamics into a low-pass filter mode to subdue the effects of the high frequency components of perturbations. 
To formulate the above, consider the introduction of a saturation function defined as

$$
\rho_{i}=\left(\tilde{u}_{i}^{u b}+\eta_{i}\right) \operatorname{sat}\left(s_{i}\right)=\left\{\begin{array}{c}
\frac{\left(\tilde{u}_{i}^{u b}+\eta_{i}\right) s_{i}}{\phi_{i}} \text { if }\left|s_{i}\right| \leq \phi_{i} \\
\left(\tilde{u}_{i}^{u b}+\eta_{i}\right) \operatorname{sgn}\left(s_{i}\right) \text { if }\left|s_{i}\right|>\phi_{i}
\end{array}\right.
$$

where $|s|=\phi$ is the so-called boundary layer. Once $\mathbf{s}$ penetrates into the boundary layer as

$$
\left|s_{i}\right| \leq \phi_{i}
$$

the $s$-dynamics takes the following form

or

$$
\begin{aligned}
& \dot{s}_{i}=\tilde{u}_{i}-\frac{\left(\tilde{u}_{i}^{u b}+\eta_{i}\right)}{\phi_{i}} s_{i} \\
& \dot{s}_{i}+\frac{\left(\tilde{u}_{i}^{u b}+\eta_{i}\right)}{\phi_{i}} s_{i}=\tilde{u}_{i}
\end{aligned}
$$

This equation represents a low-pass filter against perturbations and the tunable break frequency denoted by $\omega_{b i}$ is

$$
\omega_{b i} \equiv \frac{\left(\tilde{u}_{i}^{u b}+\eta_{i}\right)}{\phi_{i}}
$$

On the other hand, the boundary layer thickness $\phi$ is given as

$$
\phi_{i}=\frac{\left(\tilde{u}_{i}^{u b}+\eta_{i}\right)}{\omega_{b i}}
$$

The boundary layer thickness $\phi$ should be kept as small as possible for better tracking accuracy. However, larger $\eta$ offers stronger attraction of the sliding surface and hence enhanced robustness of the controller. It indicates a trade-off between performance and robustness in the design process.

Notice that the break frequency $\omega_{b}$ should be kept as large as possible because it results in thinner layer and better tracking accuracy. Moreover, even for those perturbation frequencies smaller than $\omega_{b}$, the attenuation rate is still much effective when larger $\omega_{b}$ is applied than when smaller $\omega_{b}$ is in use. Furthermore, large $\omega_{b}$ and $\eta$ can be simultaneously selected to increase the response speed of the $s$ dynamics without affecting the thickness of the boundary layer (thus affecting the tracking accuracy). The only limiting factor in the selection of $\omega_{b}$ is the control actuation frequency, which is often subject to the physical limitation of the actuator maximum actuating frequency as well as the sampling rate. From the above considerations, let

$$
\omega_{b i}=\omega_{\max i}
$$

where $\omega_{\max }$ is the limit of control actuation frequency, then replacing $\omega_{b i}$ in Eq. (102) yields

$$
\phi_{i}=\frac{\tilde{u}_{i}^{u b}+\eta_{i}}{\omega_{\max i}}
$$

A remark on the boundary layer concept is that $\widetilde{\mathbf{u}}^{u b}$ is often conservatively chosen due to the complexity and difficulty of estimating $\widetilde{\mathbf{u}}$, which may result in poor tracking accuracy (Slotine and Li 1991). This approach is often justifiable because (1) a guaranteed robustness is often 
favored over performance, (2) when the error state is outside of the boundary layer, the optimal strategy is to exercise as much torque as the actuators can afford, and (3) once the error state is pushed within the boundary layer, it is likely that the error state will not touch the boundary layer again. In this case, increasing the boundary layer has no effect on the tracking performance.

A dual-arm system is set to track a ramp input, which requires that the dual arm follow a straight line from point $(0.0)$ to $(10,10)$ with constant velocity. Figure 5 shows a trajectory plot of the end-effector of Arm 0, of Arm 1, and of the object.

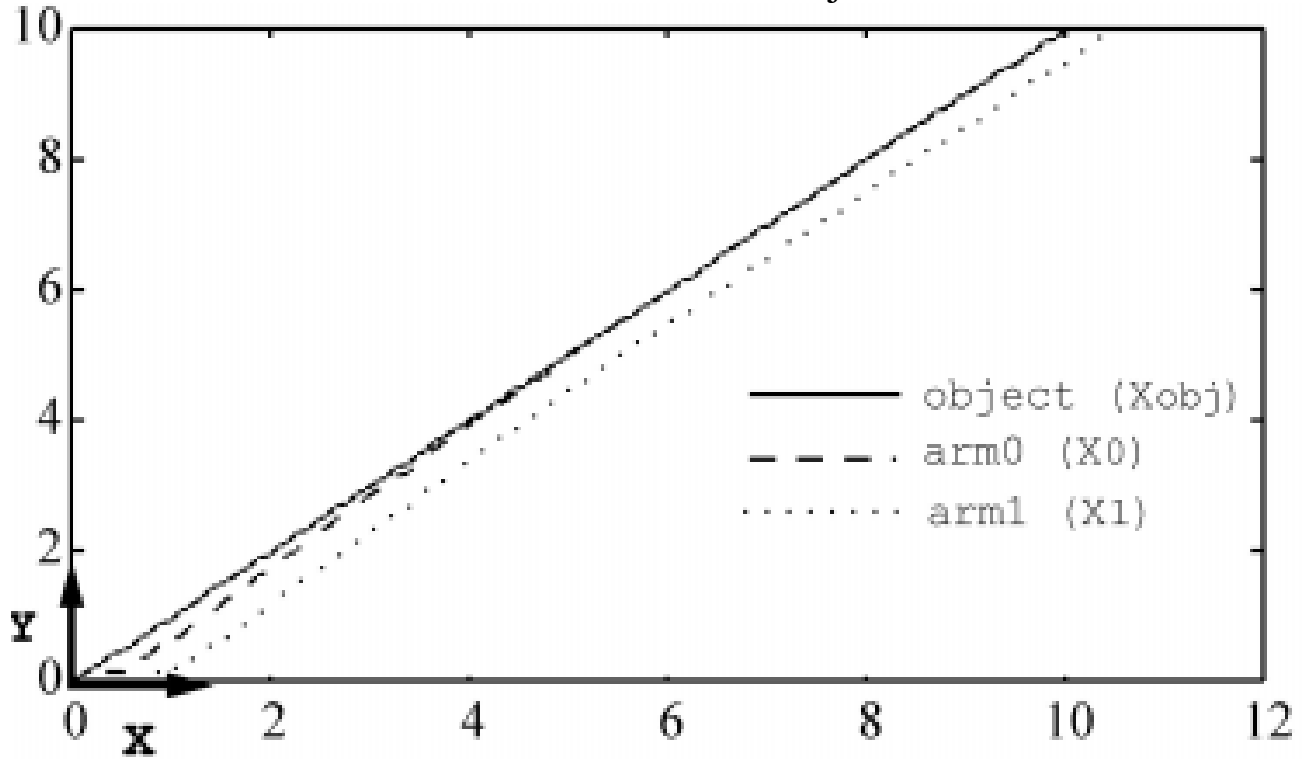

Fig. 5 Trajectory plots of the end-effector of Arm 0, Arm 1, and the object

\section{Conclusions}

Control of dual-arm cooperative systems involves vast areas of disciplines but is becoming increasingly important because of its potential use in many fields such as aerospace robotics where two floating arms are to perform orchestrated tasks; in surgery where two manipulator arms are required to perform a procedure such that an object is cooperatively manipulated; in manufacturing where the need for high level automation is becoming increasingly vital to meet the demands. The presented robust algorithm is suitable for both inertially-based problems, as well as space-based free-floating platforms.

In this project, impedance control (without external force feedback) is utilized to control individual robotic arms, where a sliding-mode control algorithm is implemented. In addition to considerable programming efforts, difficulties encountered in implementation of the proposed control method include:

(1) Estimating the upper bound of the uncertainty.

(2) Solving the K-K-T condition to obtain a force distribution vector.

(3) Selecting a proper internal force vector. 
Since the admissible solution of case 3 (used in the force distributor) may not satisfy a full set of grasp conditions, selecting appropriate values for the internal force vector becomes a key factor for successful coordination control. It requires trial-and-error procedure.

In this work, the internal force vector $\boldsymbol{\Phi}_{d}$ is assumed to have a constant given magnitude. The internal force, however, is generally a function of (1) the distance (and its derivative) between contact points and (2) the contact forces (requires a force sensor/identification). It was shown that control of the internal force is closely related to the grasp stability. Since grasp stability depends heavily on the geometry of the object and end-effectors, the control of internal force requires complicated decision making.

Solving an optimization problem with a large number of equality/inequality constraints in real time is a major computational task. While we have introduced a new methodology for dualarm grasp control, breakthroughs in this area may be possible with greater availability/reliability of automated differentiation packages.

\section{References}

Agrawal, Sunil Kumar; Shirumalla, S., 1994, "Kinematics, motion planning and design of a free-floating dual-arm planar manipulator" Mechanism \& Machine Theory v 29 n 5, pp 691-700.

Ahmadabadi, M.N. and Eiji, N., 1998, "Unified distributed cooperation strategy for multiple object handling robots", Proceedings of the 1998 IEEE International Conference on Robotics and Automation. Part 4 (of 4), v 4, Leuven, Belgium, pp.3625-3630.

Allgower, E.L. and Georg, K. 1990. Numerical Continuation Methods: An Introduction, Springer-Verlag, Berlin.

Arimoto, S. and Miyazaki, F., and Kawamura, S., 1987, "Cooperative Motion Control of Multiple Robot Arms or Fingers," IEEE International Conference on Robotics and Automation.

Arora, J.S., 1989, Introduction to Optimum Design, McGraw-Hill, Inc., New York, NY.

Bonitz, Robert G.; Hsia, T.C., 1996, "Robust dual-arm manipulation of rigid objects via palm grasping theory and experiments", Proceedings of the 1996 IEEE 13th International Conference on Robotics and Automation. Part 4 (of 4) Apr 22-28, pp 3047-3053

Cao, B; Dodds, Gordon I., and Irwin, GW., 1998, "Practical approach to near time-optimal inspection-task-sequence planning for two cooperative industrial robot arms", International Journal of Robotics Research v 17 n 8, p 858-867 0278-3649

Cheng, F. T., and Orin, O.E., 1990, "Efficient Algorithm for Optimal Force Distribution-The Compact-Dual LP Method," IEEE Transactions on Robotics and Automation, Vol. 6, No. 2.

Gardner, J.F., 1991, "Force Distribution in Walking Machines Over Rough Terrain," Transaction of ASME Journal of Dynamic Systems, Measurement, and Control, Vol. 113, No. 4, pp. 754-758.

Goldfarb, D., and Idnani, A., 1983, “A Numerically Stable Dual Method for Solving Strictly Convex Quadratic Programs,” Math. Program., Vol. 27, pp. 1-33. 
Gouo, A.; Nenchev, D.N.; Yoshida, K.; Uchiyama, M., 1998, "Dual-arm long-reach manipulators: Noncontact motion control strategies", Proceedings of the 1998 IEEE/RSJ International Conference on Intelligent Robots and Systems. Part 1 (of 3) Oct 13-17 1998 v1, p 449-454

Gu, X., Feng, C., Liu, W., Huang, Y., Jiang, X., An, G., Liu, J., and Lu, G., 1994, "Dual Arm Coordination: Model Analysis and Dynamic Compensation Control Method," Control Theory and Application, Vol. 11, No. 2, pp. 168-176.

Hogan, N. 1988, "On the stability of Manipulators Performing Contact tasks," IEEE Transactions on Robotics and Automation, Vol. 4, No. 6.

Hu, Y.R., and Goldenberg, A., 1993, "Adaptive Approach to Motion and Force Control of Multiple Coordinated Robots," ASEM Journal of Dynamic Systems, Measurement, and Control, Vol. 115, No. 1, pp. 60-69.

Hu, Y.R., and Goldenberg, A., 1993, "Dynamic Control of Coordinated Redundant Robots with Torque Optimization," Automatica, Vol. 29, No. 6, pp. 1411-1424.

Kazerooni, H. "Robust, Nonlinear Impedance Control for Robot Manipulators," Proc. IEEE Conference on Robotics and Automation., Vol 2, 1987.

Kerr, J., and Roth, B., 1986, "Analysis of Multifingered Hands," International Journal of Robotics Research, Vol 4, No. 4, Winter.

Klein, C.A., Olson, K.W., and Pugh, D.R., 1983, "Use of Force and Attitude Sensors for Locomotion of a Legged Vehicle Over Irregular Terrain," International Journal of Robotics Research, Vol. 2, No. 2, pp. 3-17.

Kumar, V., and Waldron, K.J., 1988, "Forced Distribution in Walking Vehicles," Transaction of ASME Journal of Mechanical Design, Vol. 112, No. 1, pp. 90-99.

Kwon, W. and Lee, B.H., 1998, "New optimal force distribution scheme of multiple cooperating robots using dual method", Journal of Intelligent and Robotic Systems: Theory \& Applications, v 21, n 4, p.301-326

Lee, S., 1989, "Dual Redundant Arm Configuration Optimization with Task-Oriented Dual Arm Manipulabity," IEEE Transactions on Robotics and Automation, Vol. 5, No. 1, Feb..

Li, Tsai-Yen; Latombe, Jean-Claude, 1997, "On-line manipulation planning for two robot arms in a dynamic environment" International Journal of Robotics Research, v 16 n 2, pp 144167.

Lu, W. S. and Meng, Q.H., 1992, "On Optimal Force Distribution of Coordinating Manipulators," International Journal of Robotics and Automation, Vol.7, No.2.

Montana, D. J., 1992, "Contact Stability for Two-Fingered Grasps," IEEE Transactions on Robotics and Automation, Vol. 8, No. 4.

Nahon, M. A. Angeles, J., 1992, "Optimization of Dynamic Forces in Mechanical Hands," ASME Journal of Mechanical Design, Vol. 113, June 1991.

Nahon, M.A., and Angeles, J., 1992, "Real-Time Force Optimization in Parallel Kinematic Chains under Inequality Constraints," IEEE Transactions on Robotics and Automation, Vol. 8, No. 4.

Nakamura, Y. and Ghodoussi, M., 1989, "Dynamics Computation of Closed-Link Robot Mechanisms with Nonredundant and Redundant Actuators," IEEE Transactions on Robotics and Automation, Vol. 5, No. 3, June 1989.

NASA 1995, "Resource Report," NASA TechBriefs, Vol. 19, No. 5. 
Orin, D.E., and Oh, S.Y., 1981, "Control of Force Distribution in Robotic Mechanisms Containing Closed Kinematic Chains," Transaction of ASME Journal of Dynamic Systems, Measurement, and Control, Vol. 103, pp. 134-141.

Pfeffer, Lawrence E.; Cannon, Robert H., 1993, "Experiments with a dual-armed, cooperative, flexible-drivetrain robot system", Proceedings of the IEEE International Conference on Robotics and Automation, Atlanta, GA v 3, pp 601-608

Ploen, S.R.; Park, F.C., 1997, "Lie group formulation of the dynamics of cooperating robot systems", Robotics and Autonomous Systems, v 21 n 3, Elsevier Sci B.V. Amsterdam Netherlands p 279-287.

Raven, H.H., 1995, Automatic Control Engineering, McGraw-Hill, Inc., New York, NY.

Schneider, S. A. Cannon, R. H., "Object Impedance Control for Cooperative Manipulation: Theory and Experiment Results," IEEE 1989 International Conference on Robotics and Automation.

Schneider, Stanley A.; Cannon, Robert H. Jr., 1992, “Object impedance control for cooperative manipulation: Theory and experimental results", IEEE Transactions on Robotics and Automation v 8 n 3, pp 383-394.

Tarn, T.-J.; Bejczy, A.K.; De, P.K., 1996, "Analysis of the dynamic ability of two robot arms in object handling", Advanced Robotics v10 n3 pp 301-315

Walker, I. D., Freeman, R. A., and Marcus, S. I., 1991, "Analysis of Motion and Internal Loading of Objects Grasped by Multiple Cooperating Manipulators," International Journal of Robotics Research, Vol 10, No. 4.

Yale, Gary E.; Agrawal, Brij N., 1998, "Lyapunov controller for cooperative space manipulators", Journal of Guidance, Control, and Dynamics v 21 n 3, p 477-484 